\title{
Análise estatística da qualidade das medidas das propriedades de forma de agregados com o uso do Processamento Digital de Imagens (PDI)
}

Statistical analysis of the quality of the measurements of the shape properties of aggregates using the Digital Image Processing (DIP)

\author{
Daniele Soares Ibiapina ${ }^{1}$, Lara Marques Diogenes ${ }^{2}$, Veronica Teixeira Franco Castelo Branco ${ }^{3}$, \\ Silvia Maria de Freitas ${ }^{4}$, Laura Maria Goretti da Motta ${ }^{5}$, Daianne Fernandes Diogenes ${ }^{6}$ \\ 1Universidade Federal do Ceará, Ceará - Brasil, daniele@det.ufc.br \\ 2Universidade Federal do Ceará, Ceará - Brasil, laramdiogenes@det.ufc.br \\ 3Universidade Federal do Ceará, Ceará - Brasil, veronica@det.ufc.br \\ ${ }^{4}$ Universidade Federal do Ceará, Ceará - Brasil, silvia@dema.ufc.br \\ 5Universidade Federal do Rio de Janeiro, Rio de Janeiro - Brasil, laura@coc.ufrj.br \\ 6Universidade Federal do Ceará, Ceará - Brasil, daianne@det.ufc.br
}

\section{Recebido:}

8 de outubro de 2018

Aceito para publicação:

28 de novembro de 2019

Publicado:

15 de dezembro de 2020

Editor de área:

Kamilla Vasconcelos

\section{Palavras-chaves:}

Análise estatística.

Propriedades de forma de

agregados.

Processamento Digital de Imagem (PDI).

\section{Keywords:}

Statistical analysis.

Shape properties of aggregates.

Digital Image Processing (DIP).

DOI:10.14295/transportes.v28i5.1865 OPEn 2 access

\section{RESUMO}

O Processamento Digital de Imagens (PDI) está estabelecido como ferramenta para a caracterização das propriedades de forma de agregados (forma, angularidade e textura superficial). Entre as principais técnicas de PDI, utilizadas na área de Infraestrutura de Transportes, está o uso do Aggregate Imaging Measurement System (AIMS). Este artigo tem como objetivo principal avaliar a qualidade das medidas das propriedades de forma de agregados, geradas com o uso de dois AIMS, através de análises estatísticas de repetibilidade e de reprodutibilidade. Para tanto, dois agregados de origem fonolítica, produzidos por diferentes processos de britagem, foram utilizados. Os resultados baseados em estatística descritiva e inferencial mostraram que houve repetibilidade e reprodutibilidade o que contribui na seleção de agregados, na fase de projeto, com propriedades de forma fornecidas a partir de curvas de distribuição e não por valores médios.

\begin{abstract}
The Digital Image Processing (DIP) is established as a tool for the characterization of aggregate shape properties (shape, angularity, and surface texture). Among the main techniques of DIP used in the area of Transport Infrastructure is the use of the Aggregate Imaging Measurement System (AIMS). This paper AIMS to evaluate the quality of aggregate shape properties measurements generated by the use of two AIMS, through statistical analyzes of repeatability and reproducibility. For this reason, two aggregates of phonolitic origin, produced by different crushing processes, were collected. The results based on descriptive and inferential statistics showed that there was repeatability and reproducibility, which contributes to the selection of aggregates in the project phase with shape properties provided from distribution curves and not by mean values.
\end{abstract}

\section{INTRODUCÃO}

O equipamento AIMS é utilizado para caracterizar as propriedades de forma a partir de análises de imagens 2D de partículas de agregados (Al Rousan et al., 2005; Castelo Branco et al., 2006; 
Taylor et al., 2006; Masad et al., 2007b). Esse equipamento fornece curvas de distribuição dessas propriedades para uma amostra de agregado e não somente seus valores médios, o que permitiu a elaboração de dois sistemas de classificação de propriedades de forma de agregados: americanos (Al Rousan, 2004) e brasileiros (Ibiapina, 2018).

Pesquisas internacionais conduziram a caracterização de agregados para variadas análises com a utilização do AIMS (Al Rousan, 2004; Mahmoud et al., 2010; Moaveni et al., 2014; Mahmound; Ortiz, 2014). Há várias pesquisas realizadas no Brasil, com o uso do AIMS, para análise das propriedades de forma de agregados ao longo dos últimos anos. Pesquisadores constataram a importância dessa ferramenta para as mais diversas aplicações, como por exemplo, em caracterização de: agregados para avaliar o desempenho mecânico de misturas asfálticas; agregados para avaliar a resistência à degradação; agregados para fins de comparação entre os diferentes processos de britagem; agregados para avaliar o desempenho em camada de lastro ferroviário; corpos de prova de misturas asfálticas para avaliar a textura superficial (Bessa, 2012; Alves, 2014; Pazos et al., 2015; Ibiapina et al., 2017; Diógenes et al., 2017; Diógenes, 2018). Essas pesquisas foram conduzidas com o uso do AIMS da Universidade Federal do Ceará (UFC) ou com o AIMS da Universidade Federal do Rio de Janeiro (UFRJ). Ibiapina et al. (2018) desenvolveu um sistema de classificação das propriedades de forma de agregados, caracterizados com o uso do AIMS. A classificação foi elaborada a partir de um banco de dados composto por vinte agregados, com mineralogias distintas, oriundos de diferentes localidades, abrangendo oito estados brasileiros. A autora utilizou o método estatístico de cluster, para calcular os novos valores limites dessa classificação.

Para a obtenção de resultados compatíveis entre diferentes laboratórios, programas interlaboratoriais podem ser estabelecidos (Lopes, 2010). No âmbito dos ensaios de laboratório, precisão é o termo utilizado para descrever a variação entre ensaios repetitivos (Fortes et al., 2004). A precisão tem como base as medidas de dispersão (desvio padrão e/ou Coeficiente de Variação - CV) de uma série de repetições de uma mesma análise e pode ser dividida em: repetibilidade e reprodutibilidade. Repetibilidade refere-se ao grau de concordância entre os resultados de medições sucessivas, de uma mesma grandeza, efetuadas nas mesmas condições. Reprodutibilidade refere-se às medições de uma mesma grandeza, em que as medições individuais são efetuadas variando-se uma ou mais das seguintes condições: método, operador, equipamento, instrumento de medida, local, condições de utilização e tempo (Barthem e Bertulani, 2009).

Um programa interlaboratorial consiste na organização, realização e avaliação de ensaios em produtos ou materiais idênticos ou similares, por laboratórios diferentes, sob condições prédeterminadas (ABNT ISO/IEC GUIA 43, 1999). Esse tipo de programa pode ser realizado com o objetivo de verificar a consistência dos resultados dos ensaios de um laboratório, a partir de um indicador de qualidade e da competência na realização dos ensaios (Lopes, 2010). 0 fato de um laboratório poder comparar o seu desempenho com outros laboratórios com características semelhantes contribui para a implantação de ações preventivas visando a melhoria dos seus procedimentos (INMETRO, 2009). De acordo com a ASTM E 691 (2009), os parâmetros considerados para os ensaios de laboratório são: (i) materiais: mínimo de dois materiais e suas amostras devem ser homogeneizadas; (ii) laboratórios: mínimo de cinco laboratórios participantes; (iii) operadores: um único operador é aceitável); (iv) amostras: mínimo de duas amostras de cada material analisadas por operador, em cada laboratório; (v) equipamento - este efeito não deve ser incluído na análise estatística. 
Com o objetivo de validar a metodologia proposta por Al Rousan (2004) com relação ao uso do AIMS e do Processamento Digital de Imagens (PDI) para caracterização de propriedades de forma de agregados minerais, Bathina (2005) realizou as análises de repetibilidade e de reprodutibilidade, utilizando o banco de dados gerado por Al Rousan (2004), ou seja, treze agregados graúdos e cinco agregados miúdos, de mineralogias distintas e de diferentes localidades americanas. Foram utilizadas duas frações de agregados: retidos na 9,5mm (graúda) e retidos na 1,18mm (miúda) para as análises e um único AIMS, do Texas Transportation Institute (TTI). A autora avaliou, estatisticamente, a qualidade das medidas geradas pelo AIMS baseada na norma ASTM C 802 (1996) e constatou a repetibilidade das medidas e a reprodutibilidade entre diferentes operadores.

Ibiapina (2018) elaborou um esboço de norma técnica para a caracterização de agregados com o uso do PDI. Diante disso, e do fato de não haver, ainda no Brasil, um programa interlaboratorial para esse tipo de ensaio, este artigo tem como objetivo avaliar a qualidade das medidas obtidas com o uso de dois AIMS, de laboratórios distintos. Para tanto, foi utilizado o método de análise estatística de repetibilidade e de reprodutibilidade, das medidas de propriedades de forma de agregados, desenvolvido por Ibiapina (2018).

Este artigo tem como objetivo principal avaliar a qualidade das medidas das propriedades de forma de agregados, geradas com o uso de dois AIMS, através de análises estatísticas de repetibilidade e de reprodutibilidade. Para tanto, dois agregados de origem fonolítica, produzidos por diferentes processos de britagem, foram utilizados.

\section{MATERIAIS E MÉTODOS}

\subsection{Materiais}

Os agregados utilizados para realizar as análises estatísticas de repetibilidade e de reprodutibilidade das medidas das propriedades de forma geradas com o uso do AIMS possuem origem fonolítica e foram coletados no estado do Ceará, Brasil. Os agregados processados em três estágios, no britador de mandíbulas, no britador cônico e no de impacto vertical foram denominados de material 1. Os agregados processados em um estágio, no britador de mandíbulas, foram denominados de material 2 .

\subsection{Métodos}

Inicialmente, ressalta-se que para avaliar a qualidade das medidas obtidas no AIMS foi adaptado o programa interlaboratorial da especificação ASTM E 691 (2009), com a participação de apenas dois laboratórios. Na fase experimental, utilizaram-se dois equipamentos para conduzir as análises de repetibilidade e de reprodutibilidade: o AIMS da UFC (denominado de Laboratório 1 (L1)) e o AIMS da UFRJ (denominado de Laboratório 2 (L2)). Um protocolo de utilização do equipamento foi elaborado (Ibiapina, 2018) e encaminhado para cada Operador (0) que realizou os testes. Ao todo, sete operadores realizaram três Análises (A) de cada material (denominados de material 1 e de material 2), sendo quatro operadores do L1 e três operadores do L2. Foram utilizadas duas frações: 12,5mm (graúdo) e 1,18mm (miúdo) de cada material. A Figura 1 apresenta um fluxograma do método experimental utilizado.

As análises de repetibilidade e de reprodutibilidade das medidas das propriedades de forma geradas com o uso do AIMS estão descritas a seguir: 
a) Análise de repetibilidade entre medidas: a variável cujo impacto está sendo avaliado é a variação ocorrida entre as medidas (três análises) realizadas por um mesmo operador, nas mesmas condições;

b) Análise de reprodutibilidade entre operadores: a variável cujo impacto está sendo avaliado é a variação ocorrida entre as medidas realizadas por operadores distintos (quatro operadores no L1 e três operadores no L2);

c) Análise da reprodutibilidade entre equipamentos: a variável cujo impacto está sendo avaliado é a variação ocorrida entre as medidas realizadas por operadores distintos, entre dois equipamentos distintos (AIMS do L1 e AIMS do L2).

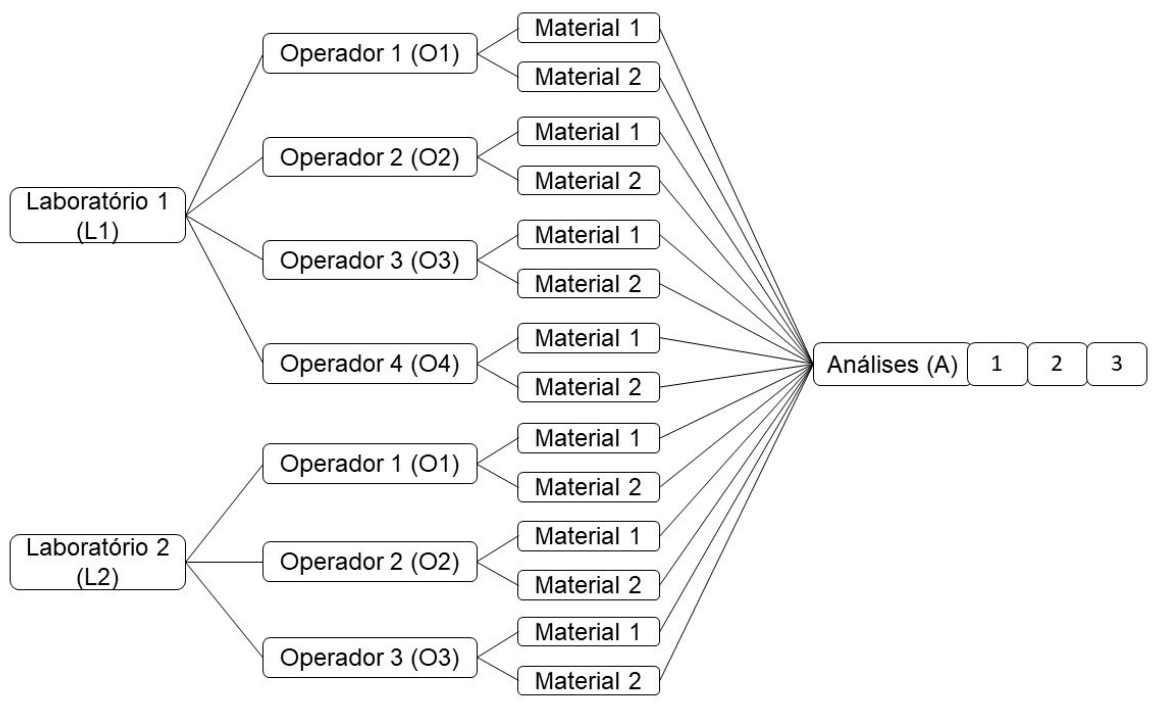

Figura 1. Fluxograma do método experimental

Após cada análise, houve reposição de material, ou seja, as partículas de agregados avaliadas foram retornadas ao recipiente que continha a amostra. Para realizar a análise seguinte, novas partículas foram selecionadas aleatoriamente (sendo um total de três análises realizadas por operador). Assim, houve influência da variabilidade da amostra, uma vez que as partículas retiradas do recipiente não foram exatamente as mesmas.

\subsubsection{Método estatístico utilizado}

O método utilizado se baseia na combinação de duas abordagens: a estatística descritiva e a estatística inferencial. Inicialmente, na abordagem descritiva, as médias foram calculadas e utilizadas nas análises como parâmetros indicadores das variações entre: cada análise (individual) de cada propriedade de forma, de cada fração e de cada material realizada por um operador; as três análises realizadas (em conjunto) por cada um dos sete operadores e as análises realizadas por todos os operadores de cada um dos dois laboratórios avaliados. Para verificar a repetibilidade e a reprodutibilidade, o critério utilizado foi o da igualdade das médias de cada propriedade de forma, de cada fração e de cada material, avaliada com a aplicação dos testes de hipótese (critério A).

$\mathrm{Na}$ abordagem inferencial, verificou-se a homogeneidade das amostras com o uso do teste de Bartlett e a normalidade dos resíduos com o uso do teste de Shapiro-Wilk, que são condições necessárias para se aplicar o teste paramétrico de análise de variâncias ANOVA Uno Way 
(um fator). 0 objetivo de se ter aplicado o ANOVA foi verificar se, pelo menos, uma análise (individual) era diferente das outras duas. Nas análises em que foram identificadas diferenças, o teste Tukey foi aplicado com o objetivo de verificar onde ocorreram essas diferenças, comparando as análises duas a duas. Para as análises que não atenderam aos pré-requisitos para a aplicação do ANOVA, aplicou-se o teste não paramétrico de Wilcoxon, com o objetivo de verificar a diferença entre análises, duas a duas. 0 nível de significância utilizado em todos os testes foi de 0,05 (Valor $\mathrm{P} \geq 0,05$ ). Os códigos dos testes aplicados, para analisar a repetibilidade entre medidas; a reprodutibilidade entre diferentes operadores e a reprodutibilidade entre diferentes laboratórios, foram elaborados por Ibiapina (2018) com o uso do software R (R CORE TEAM, 2015).

Tabela 1 - Valores limites da classificação de propriedades de forma de agregados caracterizados com o uso do AIMS

\begin{tabular}{cccccc}
\hline Propriedade & & \multicolumn{4}{c}{ Valores limites } \\
\hline \multirow{2}{*}{ Forma 2D } & $<4,0$ & $4,0-11,0$ & $11,0-15,5$ & $>15,5$ & - \\
& Circular & Semicircular & Semialongado & Alongado & - \\
\hline \multirow{2}{*}{ Angularidade } & $<1.260$ & $1.260-4.080$ & $4.080-7.180$ & $>7.180$ & - \\
& Arredondado & Subarredondado & Subangular & Angular & - \\
\hline \multirow{2}{*}{ Esfericidade } & $<0,5$ & $0,5-0,7$ & $0,7-0,9$ & $>0,9$ & - \\
& Achatado/Alongado & Baixa esfericidade & $\begin{array}{c}\text { Esfericidade } \\
\text { moderada }\end{array}$ & Alta esfericidade & - \\
\hline \multirow{2}{*}{ Textura superficial } & $<260$ & $260-440$ & $440-600$ & $600-825$ & $>825$ \\
& Polido & Macio & Baixa rugosidade & $\begin{array}{c}\text { Rugosidade } \\
\text { moderada }\end{array}$ & Alta rugosidade \\
\hline
\end{tabular}

Fonte: Ibiapina (2018)

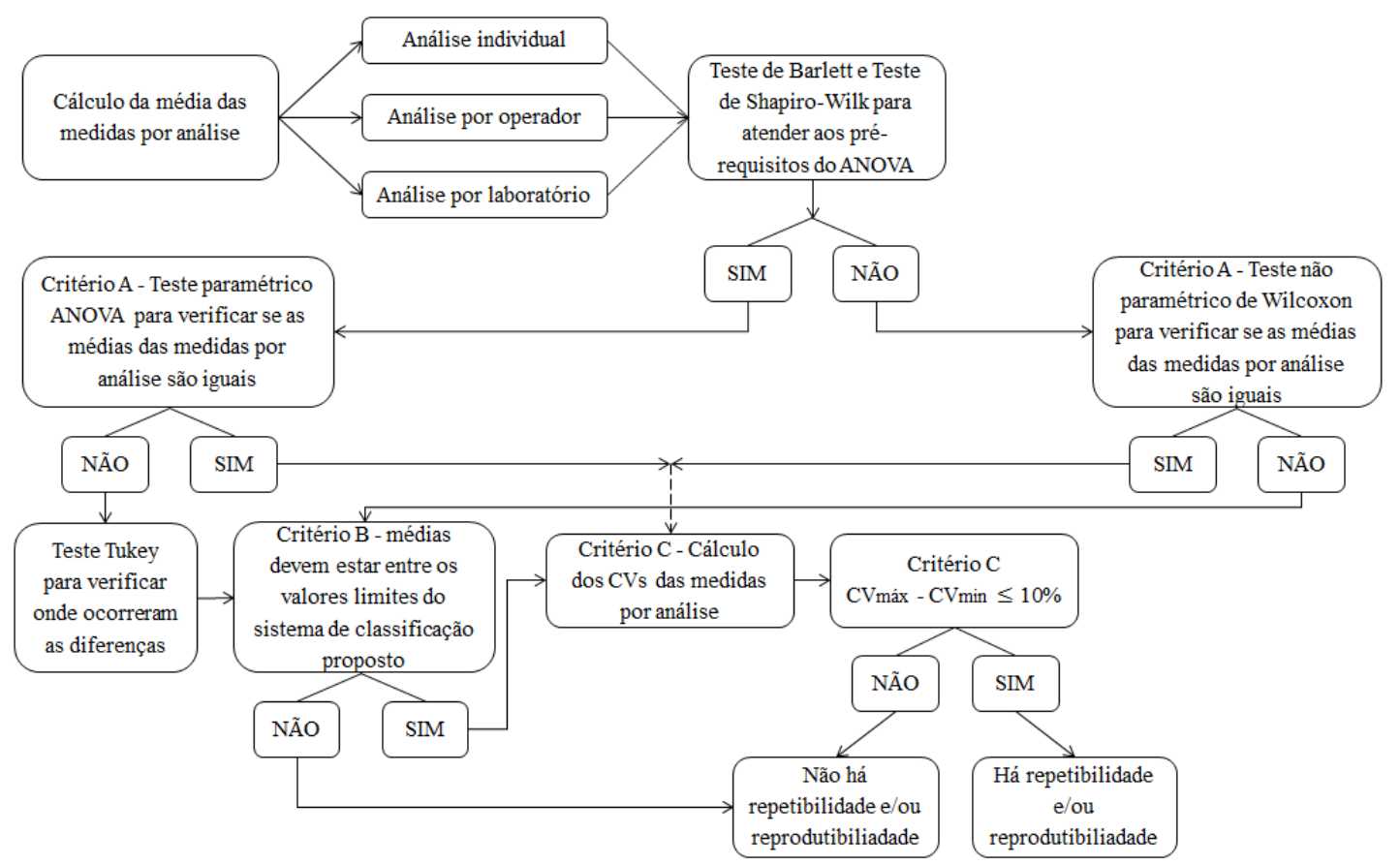

Figura 2. Fluxograma do método utilizado

Caso os resultados dos testes de hipótese não gerassem médias estatisticamente iguais, um segundo critério (critério B) de verificação de igualdade seria adotado. 0 critério B estabeleceu que essas médias fossem consideradas iguais, caso seus valores se encontrassem entre os valores que delimitassem as classes do sistema de classificação de propriedades de forma de agregados (Tabela 1). Caso os critérios A ou B fossem atendidos, os Coeficientes de Variação (CVs) 
seriam os parâmetros indicadores da variação entre as medidas. Dessa forma, o critério (critério C) para indicar a repetibilidade e a reprodutibilidade seria a consistência dos CVs, ou seja, a tendência à homogeneidade dos valores de CVs. Propôs-se a verificação dos valores de CVs mínimo e máximo de cada propriedade de forma, de cada fração e de cada material. Adotou-se o valor máximo de $10 \%$ para essa diferença (o maior valor de CV para a repetibilidade foi de $8,7 \%$ para a textura superficial e o maior valor para a reprodutibilidade foi 7,5\% para a forma 2D) e, por conseguinte, a constatação de que houve repetibilidade e/ou reprodutibilidade das medidas. A Figura 2 apresenta um fluxograma do método utilizado para testar a repetibilidade das medidas e a reprodutibilidade do AIMS.

\section{RESULTADOS E DISCUSSÕES}

\subsection{Repetibilidade entre medidas de propriedades de forma}

Os resultados dos testes de hipótese aplicados para verificar a igualdade das médias (critério A) de angularidade do L1 estão apresentados na Tabela 2. Observou-se que o valor da média (3.826) da análise 1 do 01 foi, estatisticamente, diferente das demais análises realizadas pelo mesmo operador. Para essa média diferente foi aplicado o critério B e observado que as médias das três análises se posicionaram na mesma classe de angularidade (Tabela 1). Portanto, as três médias foram consideradas iguais, sendo o próximo passo, a verificação da homogeneidade dos CVs (CVmáx - Cvmin $\leq 10 \%$ ), critério C. A Figura 3 apresenta os resultados das médias e dos CVs de angularidade do L1 e os resultados indicaram a repetibilidade entre as medidas de angularidade do L1 (critério C atendido).

Tabela 2 - Resultados das médias das medidas de angularidade por análise do L1

\begin{tabular}{|c|c|c|c|c|c|c|c|}
\hline \multirow{2}{*}{ Laboratório (L) } & \multirow{2}{*}{ Material } & \multirow{2}{*}{$\begin{array}{c}\text { Fração } \\
(\mathrm{mm})\end{array}$} & \multirow{2}{*}{ Operador (0) } & \multicolumn{3}{|c|}{ Média por análise } & \multirow[t]{2}{*}{ Classificação angularidade } \\
\hline & & & & 1 & 2 & 3 & \\
\hline \multirow{16}{*}{1} & \multirow{8}{*}{1} & \multirow{4}{*}{1,18} & 1 & $3.826 a$ & $3.466 \mathrm{~b}$ & $3.467 b$ & \multirow{16}{*}{ Subarredondado } \\
\hline & & & 2 & $4.065 a$ & $4.154 a$ & $4.255 a$ & \\
\hline & & & 3 & $3.372 \mathrm{a}$ & $3.399 a$ & $3.397 a$ & \\
\hline & & & 4 & $3.469 a$ & $3.453 a$ & $3.470 \mathrm{a}$ & \\
\hline & & \multirow{4}{*}{12,5} & 1 & $3.169 a$ & $3.064 a$ & $3.125 a$ & \\
\hline & & & 2 & $3.217 a$ & $3.124 a$ & $3.053 a$ & \\
\hline & & & 3 & $3.001 a$ & $2.994 a$ & $2.972 a$ & \\
\hline & & & 4 & $3.071 \mathrm{a}$ & $3.087 a$ & $2.880 \mathrm{a}$ & \\
\hline & \multirow{8}{*}{2} & \multirow{5}{*}{1,18} & 1 & $3.434 a$ & $3.577 a$ & $3.476 a$ & \\
\hline & & & 2 & $3.806 a$ & $3.912 \mathrm{a}$ & $3.876 a$ & \\
\hline & & & 3 & $3.582 \mathrm{a}$ & $3.522 \mathrm{a}$ & $3.537 a$ & \\
\hline & & & 4 & $3.584 a$ & $3.669 a$ & $3.521 \mathrm{a}$ & \\
\hline & & & 1 & $3.444 a$ & $3.482 \mathrm{a}$ & $3.370 \mathrm{a}$ & \\
\hline & & \multirow{3}{*}{12,5} & 2 & $3.501 a$ & $3.479 a$ & $3.366 \mathrm{a}$ & \\
\hline & & & 3 & $3.318 a$ & $3.411 a$ & $3.472 \mathrm{a}$ & \\
\hline & & & 4 & $3.535 \mathrm{a}$ & $3.331 \mathrm{a}$ & $3.252 \mathrm{a}$ & \\
\hline
\end{tabular}

Nota: Letras iguais significam médias estatisticamente iguais (Valor $\mathrm{P} \geq 0,05)$.

Observou-se, também, que as médias das medidas por análise (individual), do operador 2, do L1, do material 1, da fração 1,18mm, apesar de serem estatisticamente iguais, apresentaram valores maiores do que as demais médias. As médias das análises 2 (4.154) e 3 (4.255), do citado operador, se posicionaram na classe "subangular", ao passo que todas as outras médias se posicionaram na classe "subarredondado" (critério B atendido). Contudo, essas médias foram enquadradas na classe "subarredondado", pois as diferenças supracitadas podem ser provenientes da segregação das partículas no recipiente. 0 operador pode não ter misturado o material 
antes de selecionar, aleatoriamente, as partículas para análise ou não ter selecionado partículas provenientes de diferentes locais do recipiente. Além disso, os valores 4.154 e 4.255 mostramse superiores, respectivamente, em 1,8\% e 4,2\% ao valor do limite superior da classe "subarredondado" (4.080), sendo estes considerados percentuais irrelevantes.

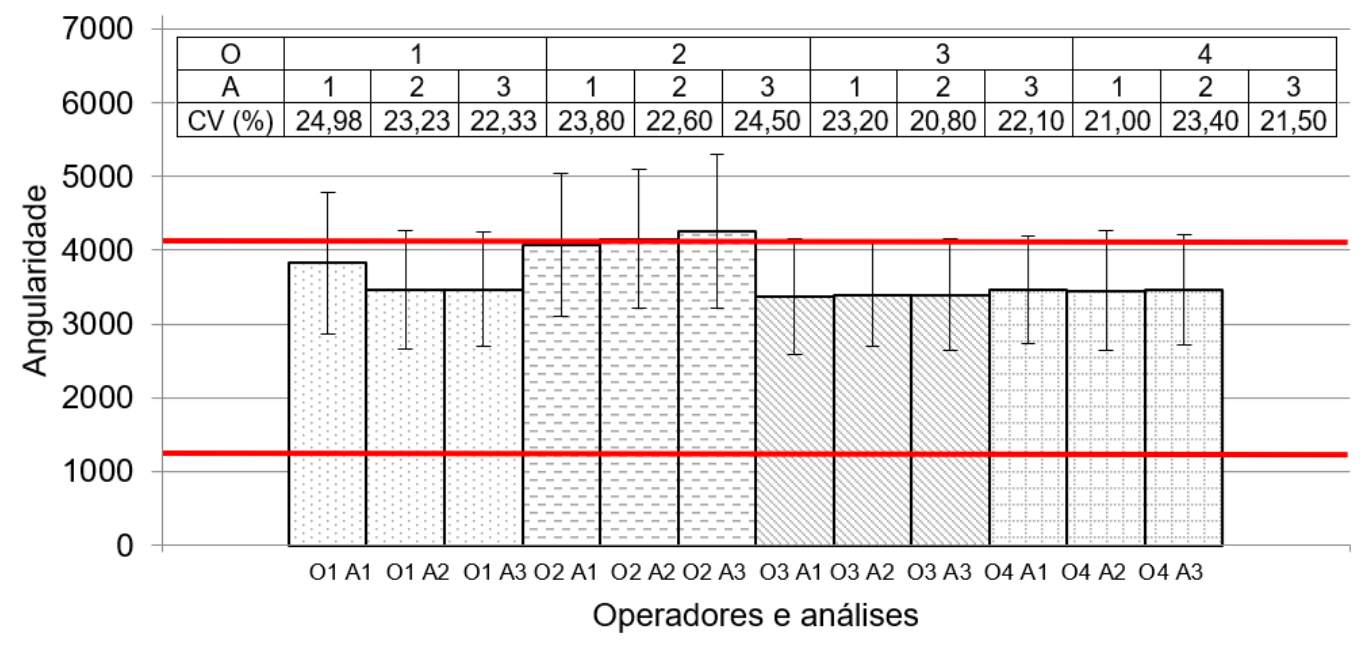

Figura 3. Resultados das médias e dos CVs de angularidade do $L 1$ do material 1 das frações miúdas $(1,18 \mathrm{~mm})$ por Operador (O) e por Análise (A)

Os resultados para verificar a igualdade das médias de angularidade do L2 estão apresentados na Tabela 3. Observou-se que o valor da média (3.179) da análise 1 do 03 foi, estatisticamente, diferente das demais análises realizadas pelo mesmo operador. Para essa média diferente, o critério B foi aplicado e atendido. A Figura 4 apresenta os resultados das médias e dos CVs de angularidade do L2, sendo constatado a repetibilidade entre as medidas de angularidade do L2 (critério $\mathrm{C}$ atendido).

Tabela 3 - Resultados das médias de angularidade por análise do L2

\begin{tabular}{|c|c|c|c|c|c|c|c|}
\hline \multirow{2}{*}{ Laboratório (L) } & \multirow{2}{*}{ Material } & \multirow{2}{*}{$\begin{array}{c}\text { Fração } \\
\text { (mm) }\end{array}$} & \multirow{2}{*}{ Operador (0) } & \multicolumn{3}{|c|}{ Média } & \multirow{2}{*}{ Classificação angularidade } \\
\hline & & & & 1 & 2 & 3 & \\
\hline \multirow{12}{*}{1} & \multirow{6}{*}{1} & \multirow{3}{*}{1,18} & 1 & $3.343 a$ & $3.439 a$ & $3.349 a$ & \multirow{12}{*}{ Subarredondado } \\
\hline & & & 2 & $3.470 a$ & $3.451 \mathrm{a}$ & $3.468 \mathrm{a}$ & \\
\hline & & & 3 & $3.391 a$ & 3.399a & $3.426 a$ & \\
\hline & & \multirow{3}{*}{12,5} & 1 & $2.878 a$ & $2.959 a$ & $3.162 \mathrm{a}$ & \\
\hline & & & 2 & $3.050 \mathrm{a}$ & $3.143 a$ & $3.136 a$ & \\
\hline & & & 3 & $3.179 a$ & $3.038 \mathrm{~b}$ & $3.029 b$ & \\
\hline & \multirow{6}{*}{2} & \multirow{3}{*}{1,18} & 1 & $3.571 a$ & $3.625 a$ & $3.512 \mathrm{a}$ & \\
\hline & & & 2 & $3.501 a$ & $3.576 a$ & $3.473 a$ & \\
\hline & & & 3 & $3.713 a$ & $3.645 a$ & $3.656 a$ & \\
\hline & & \multirow{3}{*}{12,5} & 1 & $3.456 a$ & $3.435 a$ & $3.379 a$ & \\
\hline & & & 2 & $3.566 a$ & $3.421 \mathrm{a}$ & 3.393a & \\
\hline & & & 3 & $3.681 a$ & $3.362 \mathrm{a}$ & $3.333 a$ & \\
\hline
\end{tabular}

Nota: Letras iguais significam médias estatisticamente iguais (Valor $P \geq 0,05$ )

Quanto à esfericidade e à textura superficial, os resultados das médias das medidas do L1 e do L2 atenderam ao critério A, bem como ao critério C. Portanto, constatou-se repetibilidade entre as medidas de esfericidade e de textura superficial do L1 e do L2. Quanto à classificação das propriedades de forma, as médias apresentaram para esfericidade a classe "baixa esfericidade" e para a textura superficial a classe "macio". 


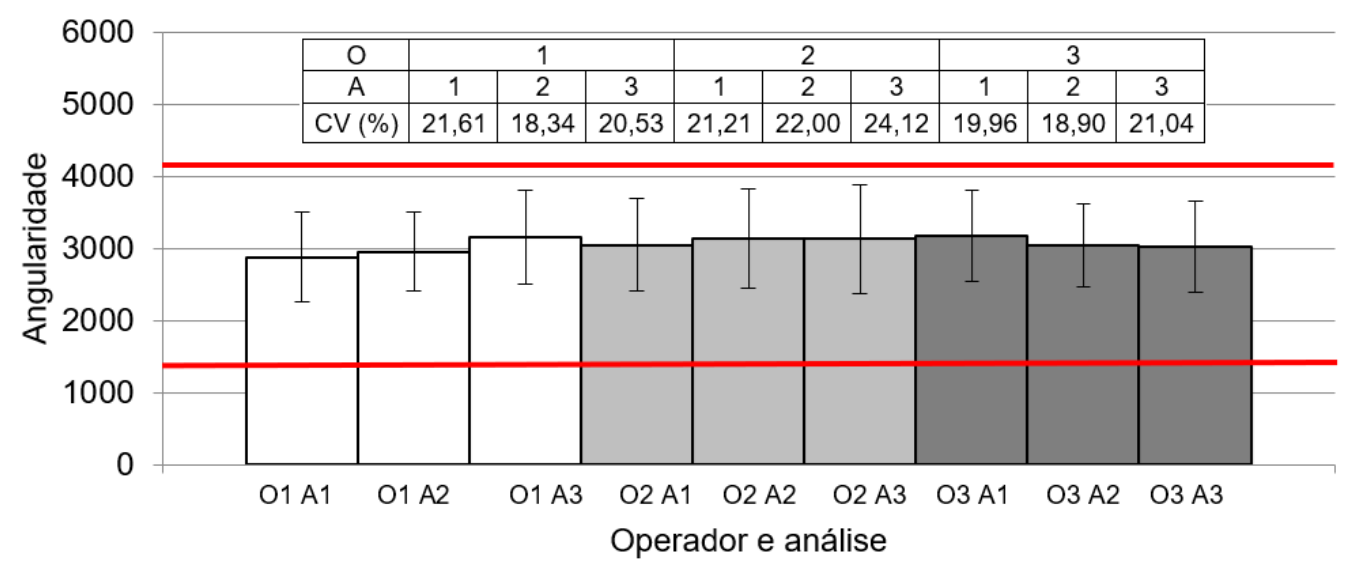

Figura 4. Resultados das médias e dos CVs de angularidade do L2 do material 1 das frações graúdas (12,5mm) por Operador (O) e por Análise (A)

Os resultados para verificar a igualdade das médias das medidas de forma 2D do L1 estão apresentados na Tabela 4. Observou-se que os valores das médias da análise 1 do $03(7,53)$, da análise 1 do 01 (7,92), da análise 1 do $02(9,05)$ e da análise 1 do $04(8,26)$ foram, estatisticamente, diferentes das demais análises realizadas pelos referidos operadores. Para as médias diferentes foi aplicado, e atendido, o critério B. Diante dos resultados dos CVs homogêneos, constatou-se a repetibilidade entre as medidas de forma 2D do L1 (critério C atendido). Com relação ao L2, as médias das medidas de forma 2D do L2 atenderam os critérios A e C. Portanto, constatou-se repetibilidade entre as medidas de forma 2D do L2.

Tabela 4 - Resultados das médias de forma 2D por análise do L1

\begin{tabular}{|c|c|c|c|c|c|c|c|}
\hline \multirow{2}{*}{ Laboratório } & \multirow{2}{*}{ Material } & \multirow[b]{2}{*}{ Fração $(\mathrm{mm})$} & \multirow{2}{*}{ Operador } & \multicolumn{3}{|c|}{ Média } & \multirow[b]{2}{*}{ Classificação forma 2D } \\
\hline & & & & 1 & 2 & 3 & \\
\hline \multirow{8}{*}{1} & \multirow{4}{*}{1} & \multirow{8}{*}{1,18} & 1 & $8,29 a$ & $8,15 a$ & $8,10 a$ & \multirow{8}{*}{ Semicircular } \\
\hline & & & 2 & $9,06 a$ & $8,65 a$ & $8,95 a$ & \\
\hline & & & 3 & $7,53 a$ & $8,05 b$ & $8,00 b$ & \\
\hline & & & 4 & $8,03 a$ & $8,20 a$ & $7,88 a$ & \\
\hline & \multirow{4}{*}{2} & & 1 & $7,92 a$ & $8,48 \mathrm{~b}$ & $8,35 b$ & \\
\hline & & & 2 & $9,05 a$ & $8,68 \mathrm{~b}$ & $8,50 \mathrm{~b}$ & \\
\hline & & & 3 & $8,16 a$ & $8,30 a$ & $8,74 a$ & \\
\hline & & & 4 & $8,26 a$ & $9,12 b$ & $8,92 b$ & \\
\hline
\end{tabular}

Nota: Letras iguais significam médias estatisticamente iguais (Valor $\mathrm{P} \geq 0,05)$

\subsection{Reprodutibilidade entre operadores}

Os resultados dos testes de hipótese aplicados para verificar a igualdade das médias de angularidade entre operadores do L1 e L2 estão apresentados na Tabela 5. Observou-se que os valores das médias das análises do 01 (3.588) e das análises do 02 no L1 (4.158) do material 1 da fração $1,18 \mathrm{~mm}$ foram diferentes entre si e diferentes das análises dos 03 e 04; os valores das médias das análises do 02 (3.865) do material 2 da fração 1,18mm foi diferente das demais análises e por fim, os valores das médias das análises do 03 (3.671) do material 2 da fração 1,18mm foi diferente das demais análises. Para essas médias diferentes foi aplicado o critério $\mathrm{B}$, sendo as médias posicionadas na mesma classe de angularidade ("subarredondado"), à exceção do valor da média das análises do 02 do L1 do material 1 da fração 1,18mm ("subangular"). Contudo, diante do fato desse valor (4.158) ser superior ao valor do limite superior da classe de angularidade "subarredondado" (4.080) em 1,9\%, o percentual foi considerado irrelevante e as 
médias foram consideradas iguais. Os resultados dos CVs foram homogêneos (critério C atendido). Portanto, constatou-se reprodutibilidade nas medidas de angularidade entre operadores do L1 e L2.

Tabela 5 - Resultados das médias de angularidade por operadores do L1 e L2

\begin{tabular}{|c|c|c|c|c|c|c|c|}
\hline \multirow{2}{*}{ Laboratório } & \multirow{2}{*}{ Material } & \multirow{2}{*}{$\begin{array}{c}\text { Fração } \\
(\mathrm{mm})\end{array}$} & \multicolumn{4}{|c|}{ Operador } & \multirow{2}{*}{ Classificação angularidade } \\
\hline & & & 1 & 2 & 3 & 4 & \\
\hline \multirow{4}{*}{1} & \multirow{2}{*}{1} & 1,18 & $3.588 a$ & $4.158 b$ & $3.390 c$ & $3.464 c$ & \multirow{8}{*}{ Subarredondado } \\
\hline & & 12,5 & 3.119a & $3.132 \mathrm{a}$ & $2.989 a$ & 3.013a & \\
\hline & \multirow{2}{*}{2} & 1,18 & $3.496 a$ & $3.865 b$ & $3.547 a$ & $3.592 a$ & \\
\hline & & 12,5 & $3.432 \mathrm{a}$ & $3.449 a$ & $3.400 \mathrm{a}$ & $3.373 a$ & \\
\hline \multirow{4}{*}{2} & \multirow{2}{*}{1} & 1,18 & $3.377 a$ & $3.463 a$ & $3.405 a$ & - & \\
\hline & & 12,5 & $3.000 \mathrm{a}$ & 3.110a & $3.082 \mathrm{a}$ & - & \\
\hline & \multirow{2}{*}{2} & 1,18 & $3.569 a$ & $3.517 a$ & $3.671 b$ & - & \\
\hline & & 12,5 & $3.423 a$ & $3.460 \mathrm{a}$ & 3.459a & - & \\
\hline
\end{tabular}

Nota: Letras iguais significam médias estatisticamente iguais (Valor $\mathrm{P} \geq 0,05)$

Os resultados dos testes de hipótese aplicados para verificar a igualdade das médias das medidas de esfericidade entre operadores do L1 e L2 estão apresentados na Tabela 6. Observouse que os valores das médias das análises do $01(0,67)$ do material 1 foram diferentes das análises dos 02, 03 e 04. Os valores das médias das análises do $03(0,62)$ do material 2 foram diferentes das análises dos 01, 02 e 04. Para essas médias diferentes foi aplicado, e atendido, o critério B. Os resultados dos CVs atenderam ao critério C, e assim, constatou-se reprodutibilidade nas medidas de esfericidade entre operadores do L1 e L2.

Tabela 6 - Resultados das médias de esfericidade por operadores do L1 e L2

\begin{tabular}{|c|c|c|c|c|c|c|c|}
\hline \multirow{2}{*}{ Laboratório } & \multirow{2}{*}{ Material } & \multirow{2}{*}{$\begin{array}{l}\text { Fração } \\
(\mathrm{mm})\end{array}$} & \multicolumn{4}{|c|}{ Operador } & \multirow{2}{*}{ Classificação esfericidade } \\
\hline & & & 1 & 2 & 3 & 4 & \\
\hline \multirow{2}{*}{1} & 1 & & $0,67 a$ & $0,68 b$ & $0,68 b$ & $0,68 b$ & \\
\hline & 2 & & $0,60 a$ & $0,61 a$ & $0,62 b$ & $0,60 a$ & \\
\hline \multirow{2}{*}{2} & 1 & 12,5 & $0,68 a$ & $0,67 a$ & $0,67 a$ & - & Baixa esfericidade \\
\hline & 2 & & $0,61 a$ & $0,61 a$ & $0,60 a$ & - & \\
\hline
\end{tabular}

Nota: Letras iguais significam médias estatisticamente iguais (Valor $\mathrm{P} \geq 0,05$ )

Os resultados dos testes de hipótese aplicados para verificar a igualdade das médias de forma 2D entre operadores do L1 e L2 estão apresentados na Tabela 7. Observou-se que os valores das médias das análises do $02(8,89)$ e das análises do 03 no L1 $(7,86)$ do material 1 foram diferentes entre si e diferentes das análises dos 01 e 04 . Os valores das médias das análises do 02 $(8,74)$ e das análises do 03 no L1 $(8,40)$ do material 2 foram diferentes entre si e diferentes das análises dos 01 e 04. Para essas médias diferentes foi aplicado, e atendido, o critério B. Os resultados dos CVs atenderam ao critério C, e assim, constatou-se reprodutibilidade nas medidas de forma 2D entre operadores do L1 e L2.

Tabela 7 - Resultados das médias de forma 2D por operadores do L1 e L2

\begin{tabular}{|c|c|c|c|c|c|c|c|}
\hline \multirow{2}{*}{ Laboratório } & \multirow{2}{*}{ Material } & \multirow{2}{*}{$\begin{array}{c}\text { Fração } \\
\text { (mm) }\end{array}$} & \multicolumn{4}{|c|}{ Operador } & \multirow{2}{*}{ Classificação forma 2D } \\
\hline & & & 1 & 2 & 3 & 4 & \\
\hline \multirow{2}{*}{1} & 1 & \multirow{4}{*}{1,18} & $8,18 a$ & $8,89 b$ & $7,86 c$ & $8,04 a$ & \multirow{4}{*}{ Semicircular } \\
\hline & 2 & & $8,25 a$ & $8,74 b$ & $8,40 b$ & $8,77 a$ & \\
\hline \multirow{2}{*}{2} & 1 & & $8,04 a$ & $8,15 a$ & $8,24 a$ & - & \\
\hline & 2 & & $8,60 a$ & $8,64 a$ & $8,80 a$ & - & \\
\hline
\end{tabular}

Nota: Letras iguais significam médias estatisticamente iguais (Valor $\mathrm{P} \geq 0,05$ ) 
Os resultados dos testes de hipótese aplicados para verificar a igualdade das médias das de textura superficial entre operadores do L1 e L2 estão apresentados na Tabela 8. Observou-se que os valores das médias das análises do 04 (365) do material 2 foram diferentes das análises dos 01, 02 e 03. Para essa média diferente foi aplicado, e atendido, o critério B. Os resultados dos CVs atenderam ao critério $\mathrm{C}$ e, portanto, constatou-se reprodutibilidade nas medidas de textura superficial entre operadores do L1 e L2.

Tabela 8 - Resultados das médias de textura superficial por operadores do L1e L2

\begin{tabular}{|c|c|c|c|c|c|c|c|}
\hline \multirow{2}{*}{ Laboratório } & \multirow{2}{*}{ Material } & \multirow{2}{*}{$\begin{array}{c}\text { Fração } \\
(\mathrm{mm})\end{array}$} & \multicolumn{4}{|c|}{ Operador } & \multirow{2}{*}{ Classificação textura superficial } \\
\hline & & & 1 & 2 & 3 & 4 & \\
\hline \multirow{2}{*}{1} & 1 & & $385 a$ & $381 a$ & $382 a$ & $371 a$ & \multirow{4}{*}{ Macio } \\
\hline & 2 & \multirow{3}{*}{12,5} & $334 a$ & $331 a$ & $337 a$ & $365 b$ & \\
\hline \multirow{2}{*}{2} & 1 & & $350 a$ & $357 a$ & $365 a$ & - & \\
\hline & 2 & & $319 a$ & $329 a$ & $315 a$ & - & \\
\hline
\end{tabular}

Nota: Letras iguais significam médias estatisticamente iguais (Valor $\mathrm{P} \geq 0,05)$

\subsection{Reprodutibilidade entre laboratórios}

Os resultados dos testes de hipótese aplicados para verificar a igualdade das médias de propriedades de forma entre laboratórios estão apresentados na Tabela 9. Observou-se que o valor da média das análises de angularidade do L2 (3.415) difere do valor da média das análises de angularidade do L1 (3.649) do material 1 da fração 1,18mm; o valor da média das análises de textura superficial do L2 (357) difere do valor da média das análises de textura superficial do L1 (380) do material 1 da fração 12,5mm e por fim, o valor da média das análises de textura superficial do L2 (321) difere do valor da média das análises de textura superficial do L1 (342) do material 2 da fração 12,5mm. Para essas médias diferentes foi aplicado, e atendido, o critério B. Diante dos resultados de que todos os CVs foram homogêneos, o critério C foi atendido e constatou-se reprodutibilidade entre os laboratórios avaliados.

Tabela 9 - Resultados das médias de propriedades de forma por laboratórios

\begin{tabular}{|c|c|c|c|c|c|}
\hline \multirow{2}{*}{ Propriedade } & \multirow{2}{*}{ Material } & \multirow{2}{*}{$\begin{array}{c}\text { Fração } \\
\text { (mm) }\end{array}$} & \multicolumn{2}{|c|}{ Laboratório } & \multirow{2}{*}{ Classificação propriedade de forma } \\
\hline & & & 1 & 2 & \\
\hline \multirow{4}{*}{ Angularidade } & \multirow{2}{*}{1} & 1,18 & $3.649 a$ & $3.415 b$ & \multirow{4}{*}{ Subarredondado } \\
\hline & & 12,5 & $3.063 a$ & $3.064 a$ & \\
\hline & \multirow{2}{*}{2} & 1,18 & $3.625 a$ & $3.586 a$ & \\
\hline & & 12,5 & $3.413 a$ & $3.447 a$ & \\
\hline \multirow{2}{*}{ Esfericidade } & 1 & \multirow{2}{*}{12,5} & $0,68 a$ & $0,67 a$ & \multirow{2}{*}{ Baixa esfericidade } \\
\hline & 2 & & $0,61 a$ & $0,61 a$ & \\
\hline \multirow{2}{*}{ Forma 2D } & 1 & \multirow{2}{*}{1,18} & $8,24 a$ & $8,14 a$ & \multirow{2}{*}{ Semicircular } \\
\hline & 2 & & $8,54 a$ & $8,68 a$ & \\
\hline \multirow{2}{*}{ Textura superficial } & 1 & \multirow{2}{*}{12,5} & $380 a$ & $357 b$ & \multirow{2}{*}{ Macio } \\
\hline & 2 & & $342 a$ & $321 b$ & \\
\hline
\end{tabular}

Nota: Letras iguais significam médias estatisticamente iguais (Valor $\mathrm{P} \geq 0,05$ )

\section{CONCLUSÕES}

A avaliação da qualidade das propriedades de forma, obtidas com o uso do AIMS, foi realizada a partir do método desenvolvido por Ibiapina (2018), para testar estatisticamente, a repetibilidade e a reprodutibilidade das mesmas. Nesse método, utilizaram-se duas abordagens: descritiva e inferencial. Na abordagem descritiva, as medidas de tendência central (média) e de 
dispersão (CV) foram utilizadas como parâmetros de análise das variações das medidas. Na abordagem inferencial, testes de hipótese foram aplicados para verificar a igualdade das médias das análises.

Ressalta-se que, no método aplicado para avaliar a qualidade das propriedades de forma de agregados, foram utilizadas as distribuições, geradas pelas análises realizadas no AIMS e não somente os valores médios das medidas, como no método proposto por Bathina (2005). Essa é uma vantagem do presente estudo, pois, por vezes, há dispersões dentro dos materiais, devido às suas características próprias encontradas para as várias frações e a representatividade pode estar comprometida. Outra vantagem desse método é a utilização da abordagem inferencial, na qual há um parâmetro objetivo para a análise dos resultados (nível de significância e Valor-P dos testes de hipótese).

No Brasil, existem três equipamentos AIMS2 em operação. Na presente pesquisa, resultados provenientes de dois equipamentos (UFC e UFRJ) foram utilizados. 0 terceiro equipamento encontra-se localizado no Centro de Pesquisa da Petrobras (Cenpes). Em suma, os resultados obtidos mostraram que as análises realizadas num equipamento AIMS são reproduzidas quando o mesmo material é caracterizado em um segundo equipamento AIMS, ou seja, estatisticamente os resultados mostraram repetibilidade entre as medidas de propriedades de forma e reprodutibilidade entre diferentes operadores e laboratórios. Essa constatação colabora para uma melhor seleção de agregados na fase de projeto baseada nas suas propriedades de forma geradas a partir de curvas de distribuição. Além disso, contribui para o desempenho e o prolongamento da vida útil dos pavimentos e, consequentemente, para a segurança do usuário e redução do investimento em execução, manutenção e recuperação de rodovias. Este estudo explorou agregados de origem fonolítica com diferentes processos de britagem. Recomenda-se, para trabalhos futuros, reproduzir a análise das medidas das propriedades de forma de agregados com diferentes mineralogias para calibrar o método utilizado.

\section{REFERÊNCIAS}

ABNT - Associação Brasileira de Normas Técnicas. ABNT ISO/IEC GUIA 43-1 (1999) Ensaios de proficiência por comparações interlaboratoriais. Brasil.

Al Rousan, T. M (2004) Characterization of aggregate shape properties using a computer automated system. Tese de Doutorado em Engenharia Civil - Texas A\&M University, College Station, Texas. Disponível em: <http://goo.gl/szfK0B>.

Al Rousan, T. M.; E. A. Masad; L. Myers e C. Speigelman (2005) New methodology for shape classification of aggregates. Journal of Transportation Research Board, v.193. DOI:103141/193-02.

Alves, V. A. H. S (2014) Avaliação da resistência à degradação mecânica de agregados da região metropolitana do Rio de Janeiro por técnicas digitais de imagens. Dissertação de Mestrado, Universidade Federal do Rio de Janeiro, Rio de Janeiro, RJ.

ASTM - American Society for Testing and Materials. ASTM C 802 (1996): Standard practice for conducting an interlaboratory test program to determine the precision of test methods for construction materials. EUA.

ASTM - American Society for Testing and Materials. ASTM E 691 (2009): Standard practice for conducting an interlaboratory study to determine the precision of a test method. EUA.

Barthen, R. e C. Bertulani (2009) Vocabulário de metrologia legal. Projeto ensino de física a distância. Instituto de Física - Universidade Federal do Rio de Janeiro (IF/UFRJ). Disponível em: <http://www.if.ufrj.br/teaching/metrol/metro.html>.

Bathina, M (2005) Quality analysis of the Aggregate Imaging System (AIMS). Dissertação de Mestrado. Texas A\&M University, College Station, Texas. Disponível em: <http://hdl.handle.net/1969.1/2416>.

Bessa, I. S (2012) Avaliação do processamento digital de imagens como ferramenta para caracterização de agregados e misturas asfálticas. Dissertação de Mestrado, Universidade Federal do Ceará, Fortaleza, CE. Disponível em:< http://www.repositorio.ufc.br/handle/riufc/4842>.

Castelo Branco, V. T. F.; E. Masad; D. N. Little; J. B. Soares e L. M. G. Motta (2006) Caracterização de forma, angularidade e textura de agregado de brita granítica e escórias de aciária usando o Aggregate Imaging System (AIMS). Anais do XX Congresso de Pesquisa e Ensino em Transportes, ANPET, Brasília, DF.

Diógenes, D. F.; R. S. Maia; V. T. F. Castelo Branco (2017) Evaluation of the ballast aggregates shape properties using digital image processing techniques. Bearing Capacity of Roads, Railways and Airfelds -Loizos et al. (Eds). Taylor \& Francis Group, London, ISBN 978-1-138-29595-7. 
Diógenes, L. M (2018) Avaliação das relações entre propriedades de agregados minerais com base nas características do processo de britagem e da rocha de origem. Dissertação de Mestrado, Universidade Federal do Ceará, Fortaleza, CE. Disponível em: > http://www.repositorio.ufc.br/handle/riufc/34523>.

Fortes, R. M.; A. Z. Neto; M. P. Assali e A. S. Barbosa Júnior (2004) Avaliação de cinco anos de programa interlaboratorial de misturas asfálticas desenvolvido no Brasil. 35a Reunião Anual de Pavimentação. Rio de Janeiro, RJ.

Ibiapina, D. S.; V. T. F. Castelo Branco; C. B. Rodrigues e I. S. Bessa (2017) Avaliacão da relacão entre angularidade de agregados e parâmetros do esqueleto mineral de misturas asfálticas. Anais do XIX CILA - Congresso Ibero Latino Americano del Asfalto. Medellin, Colômbia, 27 a 30 de novembro. Poster 317.

Ibiapina, D. S (2018) Proposição de um sistema de classificação das propriedades de forma de agregados caracterizados com o uso do processamento digital de imagens para a seleção de materiais brasileiros. Tese de Doutorado, Universidade Federal do Ceará, Fortaleza, CE. Disponível em: <http://www.repositorio.ufc.br/handle/riufc/34019>.

Ibiapina, D. S.; V. T. F. Castelo Branco; L. M. Diógenes; L. M. G. Motta e S. M. Freitas (2018) Proposição de um sistema de classificação das propriedades de forma de agregados caracterizados com o uso do processamento digital de imagens a partir de materiais oriundos do Brasil. Revista Transportes, v. 26, n. 4, p. 116-128. DOI: https://doi.org/10.14295/transportes.v26i4.1510.

INMETRO Instituto Nacional de Metrologia, Normalização e Qualidade Industrial (2009) Conheça o Inmetro. Disponível em<http://www.inmetro.gov.br/inmetro/oque.asp>. Acessado em 01/08/2017.

Lopes, M. M (2010) Programa interlaboratorial de agregados e misturas asfálticas da Rede Asfalto N/NE. Dissertação de Mestrado, Universidade Federal do Ceará, Fortaleza, CE. Disponível em: <http://hdl.handle.net/123456789/1813>.

Mahmoud, E. M.; L. Gates; E. A. Masad; S. Erdogan e E. Garboczi (2010) Comprehensive evaluation of AIMS texture, angularity, and dimension measurements. Journal of Materials in Civil Engineering, v. 22, n. 4, p. 369-379. D0I: https://doi.org/10.1061/(ASCE)MT.1943-5533.0000033.

Mahmoud, E. M. e E. Ortiz (2014) Implementation of AIMS in measuring aggregate resistance to polishing, abrasion, and breakage. Research Report no FHWA-ICT-14-014. Illinois Center for Transportation. 2014. Disponível em: <http://tti.tamu.edu/documents/5-1707-03-1.pdf>.

Masad, E. A.; V. T. F. Castelo Branco; D. N. Little e R. A. Lytton (2007b) Unified method for the analysis of controlled-strain and controlled-stress fatigue testing. International Journal of Pavement Engineering, v. 9, p. 233-246. DOI: http://dx.doi.org/10.1080/10298430701551219.

Moaveni, M; E. Mahmoud; E. M. Ortiz; E. Tutumluer e S. Beshears (2014) Use of advanced Aggregate Imaging Systems to evaluate aggregate resistance to breakage, abrasion, and polishing. Transportation Research Record: Journal of the Transportation Research Board, n. 2401, p. 1-10. DOI: https://doi.org/10.3141/2401-01.

Pazos, A. G.; F. T. Sacramento e L. M. G. Motta (2015) Efeitos de propriedades morfológicas de agregados no comportamento mecânico de misturas asfálticas. 44a Reunião Anual de Pavimentação, RAPv, Rio de Janeiro, RJ. Disponível em: <www.sinicesp.com.br/44rapv/trabalhos/TrabalhoFinal_39>.

R CORE TEAM R (2015) A language and environment for statistical computing. R Foundation for Statistical Computing, Viena, Austria. Disponível em: <https://www.R-project.org/>.

Taylor, M. A.; E. J. Garboczi; S. T. Erdogan e D. W. Fowler (2006) Some properties of irregular particles in 3D. Powder Technology, 162, 1-15. DOI: 10.1016/j.powtec.2005.10.013. 